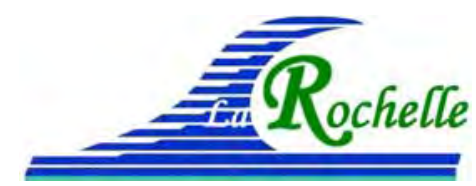

XVèmes Journées Nationales Génie Côtier - Génie Civil

La Rochelle, 29 au 31 mai 2018

DOI:10.5150/jngcgc.2018.087 ～(C) Editions Paralia CFL

disponible en ligne - http://www.paralia.fr - available online

\title{
Recherche d'optimisation pour les ouvrages de protection du littoral de Port-Neuf (La Rochelle)
}

\author{
Georges CLAVERIE ${ }^{1}$, Sébastien FILATREAU ${ }^{1}$, Caroline DEVILLIERS ${ }^{1}$, \\ Frédéric FOUAN ${ }^{2}$
}

\author{
1. CREOCEAN, 10 rue Charles Tellier, 17000 La Rochelle, France. \\ creocean@creocean.fr
}

2. SCE, Ateliers UP+, 4 rue Viviani - CS 26220, 44262 Nantes Cedex 2, France. frederic.fouan@ateliersup-plus.fr

\section{Résumé :}

Lors du passage de la tempête Xynthia, la ville de La Rochelle a été gravement touchée par des inondations. Au Nord de la baie de La Rochelle, les études du Programme d'Actions de Prévention des Inondations (PAPI) sur le secteur de Port-Neuf ont permis de montrer que le niveau extrême de la marée et l'orientation de l'agitation, lors de la tempête, étaient atypiques pour cette zone et ont été à l'origine des importantes submersions dans ce quartier de Port-Neuf. Le diagnostic du site a mis en évidence les forts enjeux vis-à-vis de la mise en sécurité des biens et des personnes, mais aussi vis-àvis du maintien des agréments de cette corniche littorale qui offre notamment un lieu de promenade très fréquenté, par les Rochelais et les touristes, et bénéficie d'une très belle vue sur l'ensemble de la baie de La Rochelle et sur le pertuis d'Antioche.

Pour réaliser une protection permettant de mettre en sécurité les biens et les personnes l'étude a montré qu'une protection côtière "conventionnelle" (digue à talus en enrochement) s'avérait beaucoup trop haute et ne permettait pas de conserver les vues sur l'espace maritime. La recherche de scénarios d'aménagement a abouti à un système de protection assez novateur consistant à réaliser, d'une part, un premier ouvrage relativement bas, autorisant des franchissements importants par paquets de mer et, d'autre part, un muret à une dizaine de mètres en retrait afin de contenir ces masses d'eau. Le muret arrière et l'ouvrage frontal formeront un canal qui permettra d'évacuer les eaux vers une zone moins agitée (l'anse de Port-Neuf).

Lors des phases d'alerte vagues/submersions, l'ouvrage sera interdit au public mais, le reste du temps, le canal, pensé comme un élément de réaménagement de l'espace, sera un lieu de promenade et permettra de mettre en valeur le site grâce à des aménagements qualitatifs. Ce scénario est apparu comme un très bon compromis entre un niveau de protection acceptable, un impact modéré dans le paysage et une emprise raisonnable.

Mots-clés :

Hydrodynamique maritime, Modèle numérique, Submersion, Inondation, Ingénierie côtière, Ouvrages de protection des côtes, Travaux maritimes, Environnement littoral. 


\section{Thème 7 - Risques côtiers}

\section{Introduction}

Lors du passage de la tempête Xynthia, le 28 février 2010, la ville de La Rochelle a été gravement touchée par des inondations, révélant la faiblesse et (ou) la trop faible hauteur des ouvrages de défenses contre la mer (voir figure 1). Une réflexion globale, portée à tous les niveaux institutionnels, s'en est suivie pour constater la nécessité de revoir les niveaux de risque et réadapter l'ensemble du dispositif de protection du littoral. Ainsi, l'agglomération rochelaise a élaboré son Programme d'Actions de Prévention des Inondations (PAPI), pour déterminer sa stratégie en termes de prévention/prévision et en termes de protection.

Au nord de la baie de La Rochelle, le niveau extrême de la marée et l'orientation sudouest de l'agitation, lors de la tempête, étaient atypiques pour cette zone et ont été à l'origine des importantes submersions dans le quartier de Port-Neuf.

Le littoral de Port-Neuf, autrefois le débouché naturel d'un marais qui s'est lentement refermé, a été progressivement recouvert de blocs d'enrochements sans que ceux-ci ne constituent une véritable protection. Il représente un linéaire de 1650 mètres. Au centre de ce linéaire, le trait de côte est resté plus naturel. Il s'agit d'une petite anse moins exposée mais dont la protection est renforcée par une digue qui a permis au cours du XXème siècle de développer une activité industrielle utilisant une cale de mise à l'eau.

Tout le linéaire a fait l'objet d'un aménagement qualitatif pour les piétons et deux roues. En arrière, le quartier de Port-Neuf s'est largement développé en limite du marais et de la mer, sans réelle prise en compte du risque lié à la proximité de l'océan.

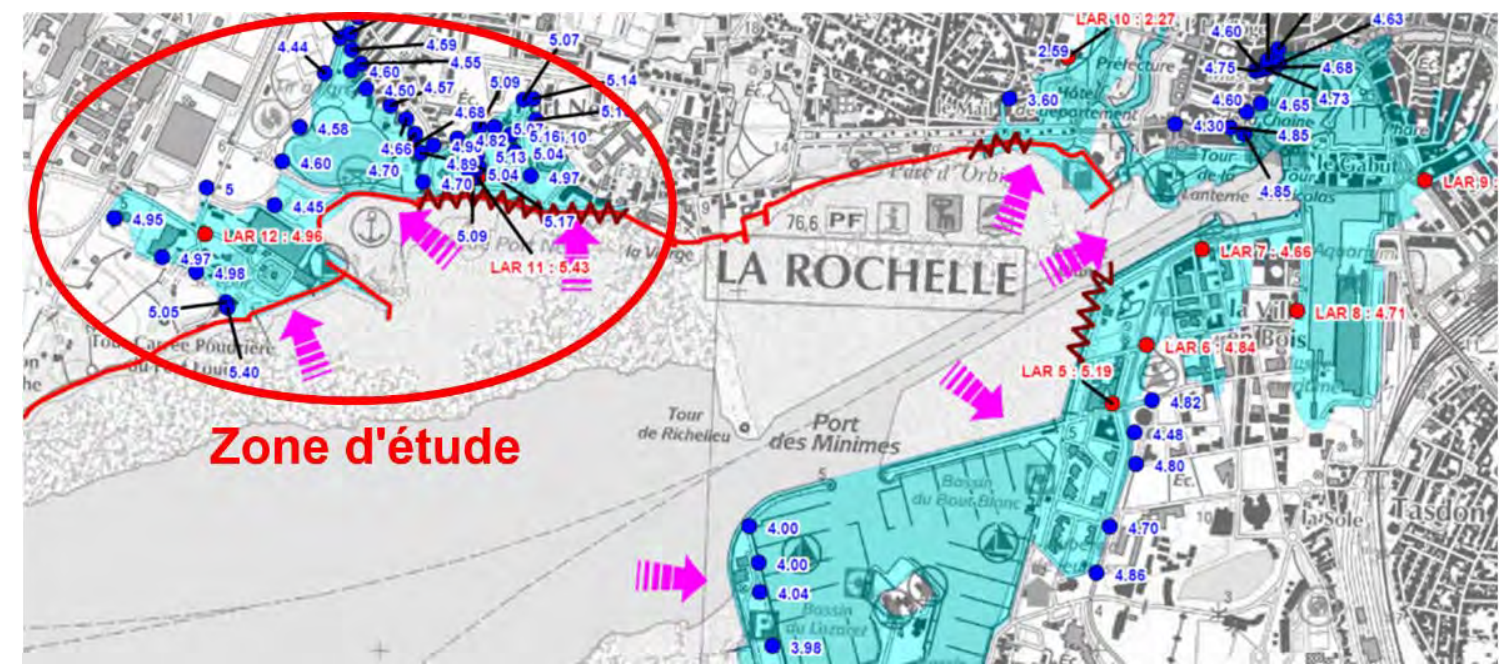

Figure 1. Visualisation des submersions sur la zone de Port-Neuf (REX DDTM17).

Le diagnostic du site a mis en évidence les forts enjeux de mise en sécurité des biens et des personnes (écoles, établissements de santé, habitations, STEP, ...) mais aussi de maintien des qualités de ce secteur urbain classé en Zone de Protection du Patrimoine Architectural, Urbain et Paysager (ZPPAUP), qui offre notamment un lieu de 


\section{XVèmes Journées Nationales Génie Côtier - Génie Civil \\ La Rochelle, 29 au 31 mai 2018}

promenade, très fréquenté par les rochelais et les touristes, et permet une très belle échappée visuelle sur l'ensemble de la baie de La Rochelle et sur le pertuis d'Antioche. Pour, dans un premier temps, les assister pour le montage du dossier PAPI puis, dans un deuxième temps, concevoir les ouvrages dans le cadre d'une maîtrise d'œuvre (incluant l'ensemble des dossiers environnementaux et réglementaires), la ville de La Rochelle a fait appel à la société CREOCEAN, en groupement avec la société SCE (deux filiales du groupe KERAN), pour rechercher les possibilités de protections puis concevoir un aménagement prenant en compte les contraintes techniques et environnementales mais répondant aussi aux exigences qualitatives qu'imposent ce site et ses usages.

\section{Etudes réalisées, recherche de solutions}

Dans le cadre de la mission, les volets d'étude sont nombreux (dont notamment les aspects environnementaux, la limitation des impacts, la concertation, les aspects réglementaires, ...) et ne pourront pas tous être présentés. Dans le présent article, les auteurs se sont concentrés sur les éléments permettant d'aboutir à un système de protection relativement novateur.

\subsection{Etudes hydrodynamiques}

Les études hydrodynamiques ont fait appel à des modélisations numériques réalisées à l'aide des logiciels développés par DHI : MIKE 3D HD FM (courant et niveau), couplé avec le module MIKE $21 \mathrm{SW}$ (génération et propagation de l'agitation). Basées sur l'utilisation de différents modèles imbriqués (incluant notamment pour l'un d'eux l'atlantique Nord-Est afin de bien prendre en compte les variations du niveau marin dues aux phénomènes météorologiques), les modélisations sont forcées avec les données les plus proches des conditions Xynthia : le vent et la pression atmosphérique (variable dans le temps et sur le domaine : résultats de modèles opérationnels, dont ECMWF, Aladin et Arome), la houle au large (résultats du modèle Hindcast WaveWatch3 (NOAA)), la marée (harmoniques du SHOM) (voir l'exemple du modèle "golfe de Gascogne" en figure 2).

Après calage de l'ensemble du modèle, ce dernier a été validé à partir des mesures existantes (marégraphes de la côte atlantique, mesures de houle au large de l'île d'Oléron). Les résultats ont montré une très bonne corrélation avec l'ensemble des résultats mesurés pendant la tempête Xynthia et ont donc permis de bien caractériser l'évolution de la surcote pendant la tempête ainsi que les évolutions de hauteur et de direction de la houle.

La figure 3 synthétise les niveaux atteints pendant la tempête Xynthia en visualisant, dans les pertuis autours des îles de Ré et d'Oléron, la hauteur maximale atteinte en chaque point du modèle durant la simulation. 
Thème 7 - Risques côtiers

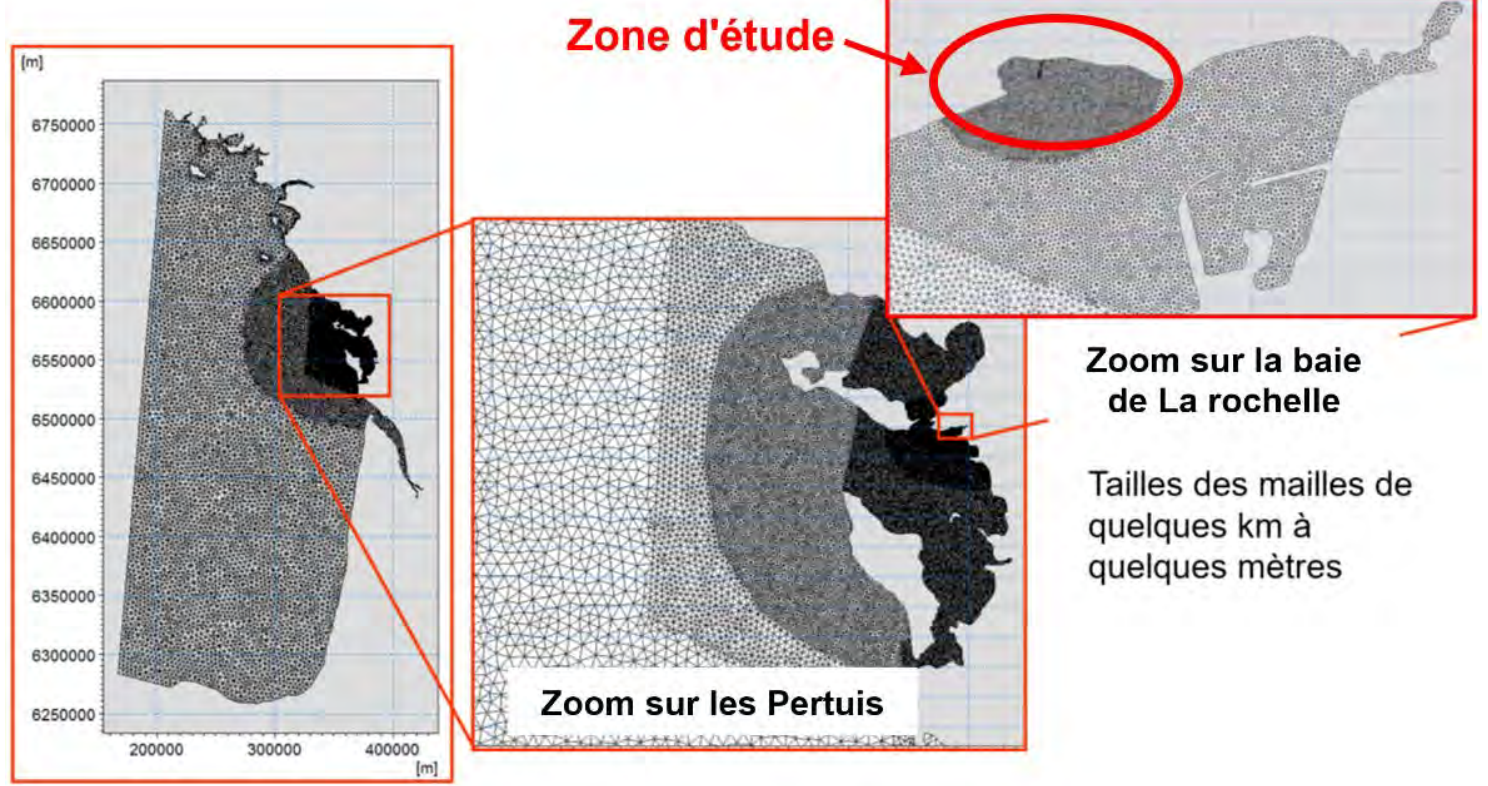

Figure 2. Maillage du modèle "golfe de Gascogne".

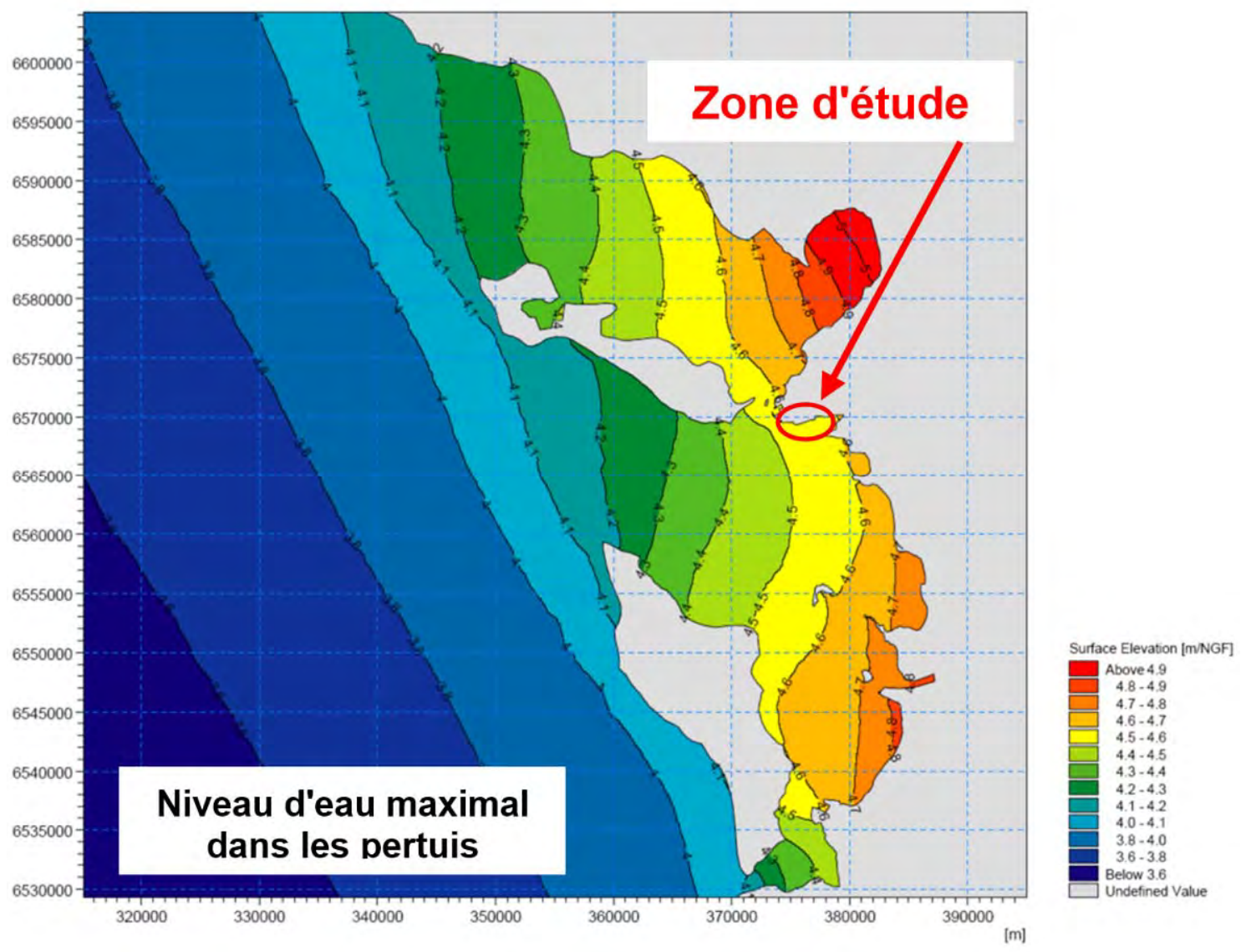

Figure 3. Modélisation du niveau d'eau en conditions Xynthia.

Visualisation du maximum atteint dans les Pertuis. 


\section{XVèmes Journées Nationales Génie Côtier - Génie Civil \\ La Rochelle, 29 au 31 mai 2018}

Compte tenu de la marée de vive eau et du phénomène de surcote (niveau extrême d'environ 4,50 m NGF mesuré au port de La Pallice (environ $8 \mathrm{~m}$ Cote Marine)) et de la direction atypique de la tempête (orientation de Sud-Ouest lors de l'intensité maximale sur la zone d'étude), la houle a atteint la côte nord de la baie de la Rochelle avec une intensité exceptionnelle (Hs supérieure à $2 \mathrm{~m}$ aux abords de la côte). (Voir figure 4).

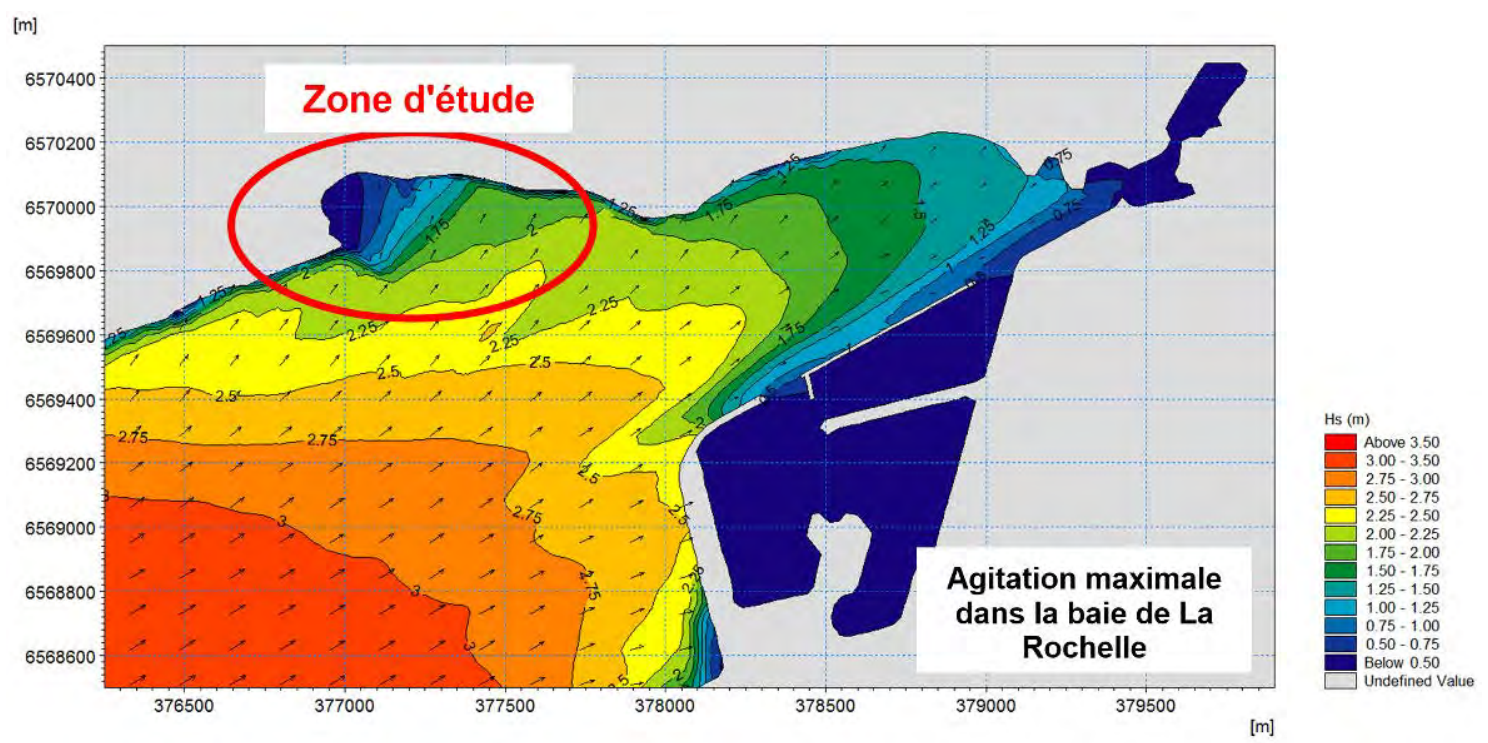

Figure 4. Modélisation de l'agitation (Hs) en conditions Xynthia. Visualisation du maximum atteint dans la baie de La Rochelle,

Pour les besoins de l'étude, afin d'anticiper l'effet du réchauffement climatique, de nouvelles simulations ont été réalisées en surélevant de $20 \mathrm{~cm}$ le niveau du plan d'eau au repos. Ces simulations ont permis de définir les conditions de projet Xynthia $+20 \mathrm{~cm}$.

\subsection{Recherche de solutions de protection}

La recherche de solutions a été réalisée de manière relativement exhaustive. La délocalisation des enjeux, les protections individuelles, la protection rapprochée des enjeux et une protection du trait de côte "conventionnelle" ont été analysées mais n'ont pas permis d'apporter une réponse satisfaisante.

La difficulté de protection de cette zone résulte du fait que le terrain naturel du trait de côte actuel est très bas avec des ouvrages sous-dimensionnés (voir figure 5a), ce qui entraine des débits extrêmement élevés en conditions de projet. Pour atteindre le niveau de protection souhaité (débit moyen maximal franchissant les ouvrages de 1 litre/s par mètre linéaire d'ouvrage), une digue à talus "conventionnelle" en enrochement aura une hauteur de plus de 2 mètres par rapport aux zones de circulations actuelles et ne permettra plus de points de vue vers la mer (voir figure 5b). 


\section{Thème 7 - Risques côtiers}

La recherche de scénarios, prenant en compte les besoins, les enjeux et les différentes contraintes du site ont conduit, parmi les aménagements réalisables, à proposer un système de protection additionnant des ouvrages de faible hauteur (voir figure $5 \mathrm{c}$ ) :

- Un premier ouvrage frontal relativement bas, constitué par une digue à talus en enrochement avec un mur de couronnement, permettant d'atténuer l'énergie de la houle mais impliquant des franchissements importants par paquets de mer.

- Un canal, assez large pour ralentir la vitesse et l'énergie des paquets de mer, permettant de contenir puis de renvoyer les volumes franchissant vers la mer.

- Un muret arrière, limitant les débits vers la zone terrestre dans une gamme acceptable.
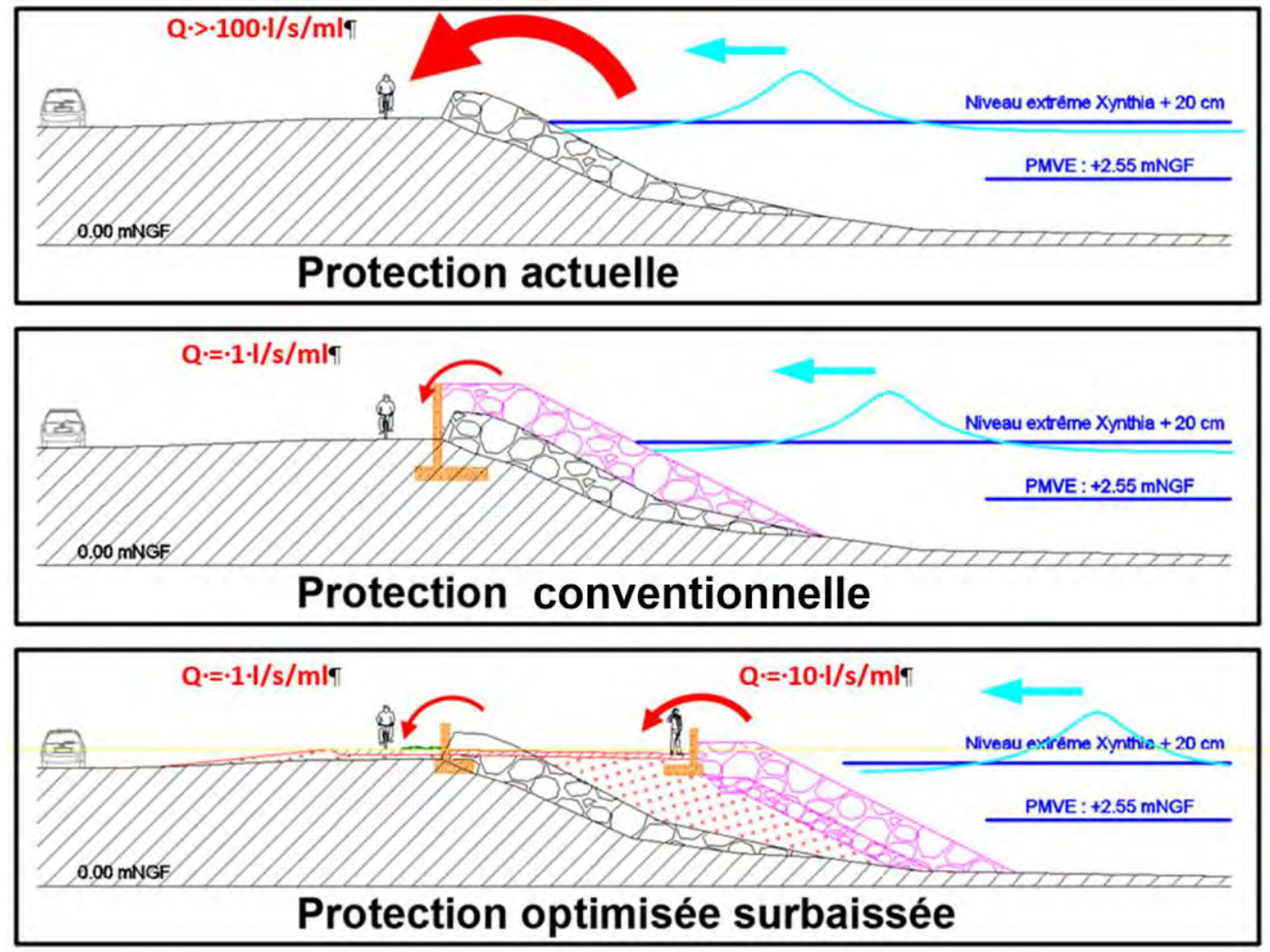

Figure 5. Profils types de la protection devant Port-Neuf : $5 a$ : Ouvrage actuel (haut), $5 b$ : Scénario non retenu d'une protection conventionnelle (milieu), $5 c$ : Ouvrages optimisés proposés (bas).

Pour être pleinement opérationnel, le canal doit pouvoir s'écouler vers une zone basse, relativement protégée de la houle afin que les vagues déferlantes ne freinent pas la vidange. Sur le site étudié, la zone centrale de l'anse de Port-Neuf peut permettre l'écoulement gravitaire vers la mer.

Cette association d'ouvrages, dès la conception initiale de l'aménagement, est apparue comme relativement novatrice en raison du peu d'exemples recensés dans la bibliographie. 


\section{XVèmes Journées Nationales Génie Côtier - Génie Civil \\ La Rochelle, 29 au 31 mai 2018}

\section{Scénario retenu}

\subsection{Principes}

La définition d'une protection optimisée surbaissée, non conventionnelle, a fait l'objet d'une attention soutenue des services de l'État (DDTM, DREAL, ABF) pendant les études d'esquisses et d'avant-projet afin de s'assurer que le projet corresponde bien aux objectifs du PAPI et soit compatible avec les contraintes du site. Pendant toutes ces études, le maître d'ouvrage a été attentif au fait que le projet ne se limite pas seulement à des ouvrages techniques mais soit une protection pensée comme un élément de réaménagement de l'espace, permettant de le valoriser. Cette démarche se retrouve donc en accord avec le récent "Guide méthodologique appliqué aux sites classés" (MTES, 2017).

Différentes optimisations techniques ont donc été recherchées (largeur du canal, hauteur des ouvrages, emprise sur la mer, débits et volumes admissibles, ...), tout en essayant de caractériser au mieux le fonctionnement hydraulique de l'ensemble des ouvrages. Les résultats ont montré que l'ouvrage envisagé initialement était globalement adapté. Les principales optimisations, par rapport à la solution envisagée dans le PAPI de 2012, ont permis de limiter les caractéristiques de la carapace en enrochement et de limiter la hauteur des murets (variable entre 0,5 et $1 \mathrm{~m} / \mathrm{sol}$ fini). La coupe type visualisée sur la figure 5c est la version optimisée de 2017. L'implantation des ouvrages a aussi été optimisée dans son tracé. L'anse de Port-Neuf sera protégée par une digue à talus "conventionnelle" et sera le lieu du débouché des canaux des "protections optimisées surbaissées" situées à l'est et à l'ouest (voir figure 6).

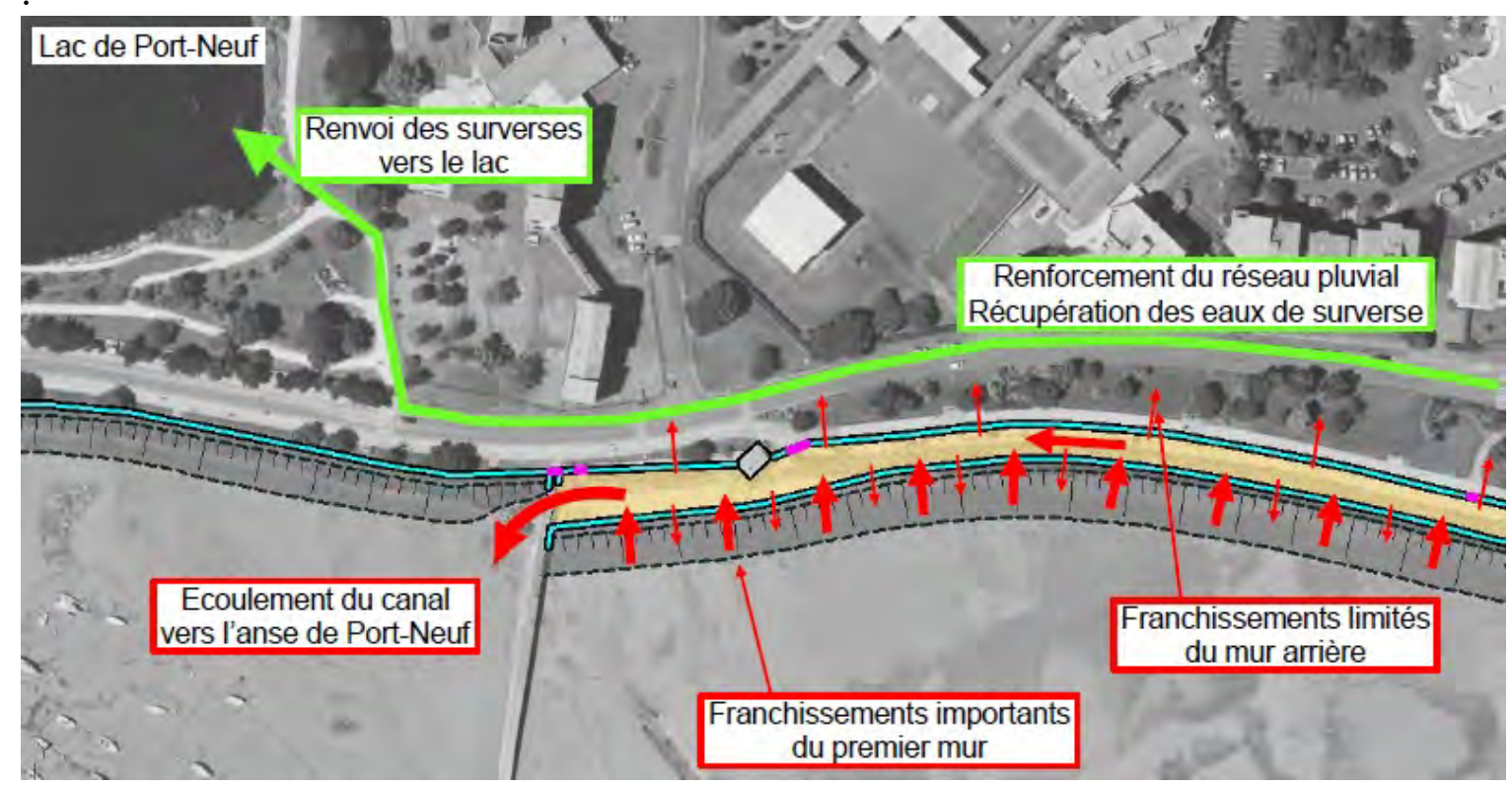

Figure 6. Plan masse, niveau esquisse.

Zoom sur la partie rejet des eaux du canal Est vers l'anse de Port-Neuf. 


\section{Thème 7 - Risques côtiers}

\subsection{Dimensionnement des ouvrages}

Le dimensionnement de ce nouveau type d'ouvrage a été fait sur la base des documents les plus récents, notamment le "Guide Enrochement" (CIRIA et al., 2009) et "l'EurOtop Manual" (EurOtop, 2016), et une recherche de l'état de l'Art pouvant s'appliquer sur la configuration retenue. Un ouvrage reposant sensiblement sur les mêmes principes a notamment été réalisé à Ostende (Belgique) et a fait l'objet d'une thèse, incluant des modélisations physiques (GEERAERTS et al., 2006), (GYSENS et al., 2011), (VAN DOORSLAER et al., 2011). Malheureusement, l'ouvrage frontal étudié pour Ostende (perré maçonné) a des caractéristiques hydrauliques trop éloignées d'une digue à talus en enrochement (telle qu'envisagée pour Port-Neuf) pour que les formulations de calcul pour les ouvrages d'Ostende puissent s'appliquer sur le cas présent.

Le débit franchissant l'ensemble de l'ouvrage a donc été évalué en décomposant les ouvrages et en prenant notamment en compte : le débit franchissant le premier ouvrage, le niveau d'eau induit dans le canal, l'agitation induite dans le canal, puis les franchissements sur le deuxième muret. Les différents calculs ont été réalisés à partir des recommandations de "l'EurOtop Manual" (EurOtop, 2016). Différentes hypothèses simplificatrices ont été faites sur ce calcul du débit franchissant global, tout en essayant de rester dans des approximations sécuritaires. Pour tenir compte des contraintes s'imposant au maitre d'ouvrage, il a été décidé de ne pas planifier d'optimisation basée sur des modélisations physiques de l'ouvrage en raison des délais importants que cela aurait nécessité, retardant ainsi la réalisation des ouvrages.

Parallèlement, la conception de l'ouvrage et sa stabilité ont été étudiées, notamment en s'appuyant sur les recommandations du "Guide Enrochement".

En conséquence, même si l'aménagement n'a pas fait l'objet de modélisations physiques pour optimiser le débit franchissant l'ensemble de l'ouvrage, la conception de l'ouvrage s'est fortement appuyée sur les multiples modélisations physiques réalisées pour établir le "Guide Enrochement" (CIRIA et al., 2009) et "l'EurOtop Manual" (EurOtop, 2016), tout particulièrement pour le premier ouvrage de protection (talus en enrochements et mur de couronnement).

\subsection{Modélisation des submersions}

La modélisation des submersions a été faite en tenant compte de l'évolution au cours du temps des caractéristiques du niveau de la mer et de la houle en pied d'ouvrage mais aussi de l'évolution des conditions hydrauliques dans le canal et au pied du muret arrière.

Cela a permis d'optimiser les ouvrages en tolérant des débits supérieurs à ceux envisagés initialement, tout en respectant un volume total admissible au cours de la tempête (voir figure 7). Sur certaines zones, un ressuyage vers le réseau pluvial a été prévu. 


\section{XVèmes Journées Nationales Génie Côtier - Génie Civil \\ La Rochelle, 29 au 31 mai 2018}

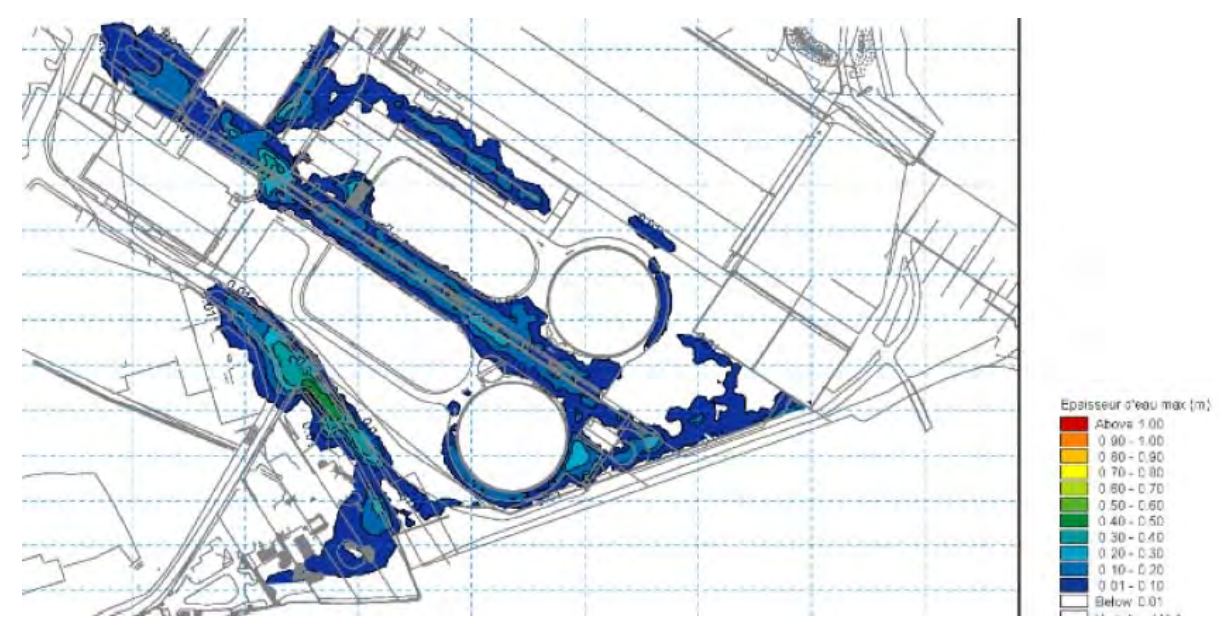

Figure 7. Modélisation des submersions futures en condition Xynthia+20cm. Zone de la station d'épuration. Epaisseur d'eau maximale.

\subsection{Aménagements qualitatifs}

Tout au long de l'étude, les caractéristiques de l'ouvrage ont été définies pour privilégier la protection contre les submersions. Néanmoins, il est rapidement apparu que le canal, au-delà de la protection qu'il offre en condition de tempête, pouvait être défini comme un lieu de promenade, offrant de nombreux points de vue vers la mer, et pouvait être mis en valeur grâce à des aménagements qualitatifs (voir figure 8).

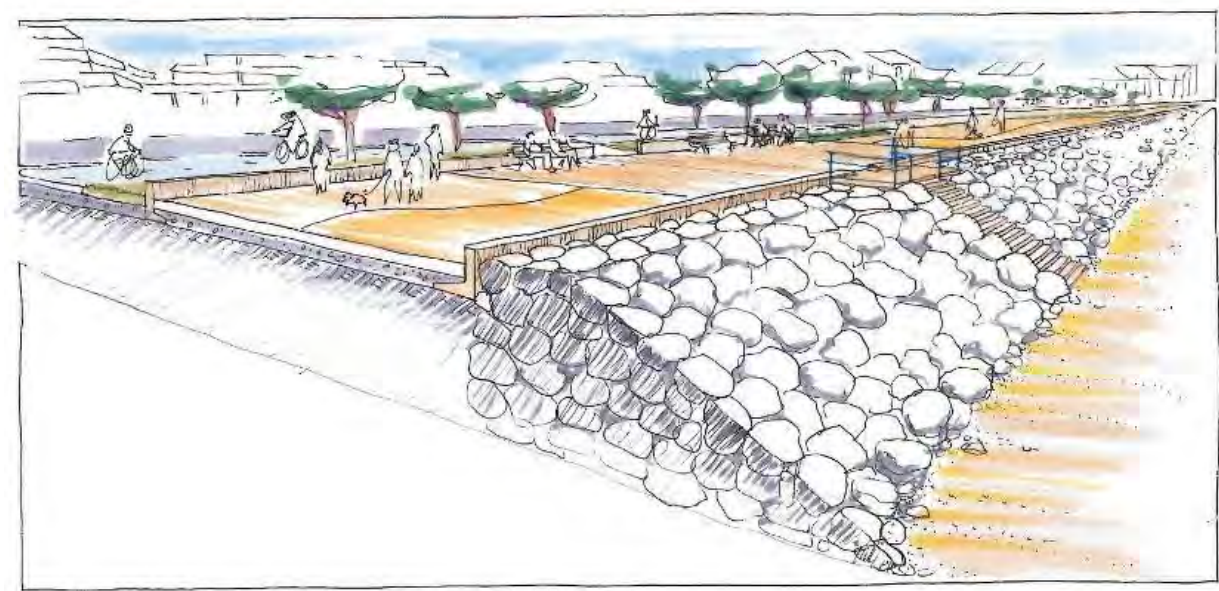

Figure 8. Visualisation des aménagements le long du Bd. W Churchill.

Tout en restant limités pour ne pas modifier les caractéristiques hydrauliques du canal, des plantations, des bancs, des accès vers la mer, ... sont prévus. Ils seront associés à différents partis pris pour les choix des enrochements, les finitions des bétons (fond du canal et murets), pour créer différentes ambiances et casser l'aspect trop monotone du linéaire important des ouvrages et également pour respecter les contraintes paysagères de la ZPPAUP. 


\section{Thème 7 - Risques côtiers}

\section{Conclusions}

Le système de protection relativement novateur envisagé a nécessité des études détaillées pour justifier les caractéristiques de l'ouvrage. Cela étant, ces études ont aussi permis d'optimiser la conception des ouvrages et ainsi limiter leurs impacts. Parallèlement, le projet a été pensé comme un élément de réaménagement de l'espace afin qu'il s'insère au mieux dans le site et permette de le valoriser. Lors des phases d'alerte aux vagues/submersions, l'ouvrage sera interdit au public mais, le reste du temps, le canal sera un lieu de promenade, avec de nombreux aménagements qualitatifs, permettant sa mise en valeur. Ce scénario est apparu comme un très bon compromis entre un niveau de protection acceptable, un impact modéré dans le paysage et une emprise raisonnable. (Voir résultat de la concertation : //www.larochelle.fr/cadre-devie/grands-projets-en-cours/papi/papi-port-neuf.html). Le dossier de demande d'autorisation pour réaliser cet ouvrage devrait être déposé en préfecture au cours du printemps 2018 .

\section{Références bibliographiques}

CIRIA, CUR, CETMEF (2009). Guide Enrochement. L'utilisation des enrochements pour les ouvrages hydrauliques. Version française du Rock Manual. CETMEF, Compiègne.

EurOtop - VAN DER MEER J.W., ALLSOP N.W.H., BRUCE T., DE ROUCK J., KORTENHAUS A., PULLEN T., SCHÜTTRUMPF H., TROCH P., ZANUTTIGH B. (2016). Manual on wave overtopping of sea defences and related structures. An overtopping manual largely based on European research, but for worldwide application. URL : www.overtopping-manual.com.

GEERAERTS J., DE ROUCK J., BEELS C., GYSENS S., DE WOLF P. (2006). Reduction of wave overtopping at seadikes SWB. Proceedings of the 30th International Coastal Engineering, pp 4680-4691.

GYSENS S., DE ROUCK J., TROUW K., BOLLE A., WILLEMS M. (2011). Integrated coastal and maritime plan for Oostende design of soft and hard coastal protection measures during the EIA procedures. Coastal Engineering Proceedings, 1(32), management-37, $10 \mathrm{p}$. https://doi.org/10.9753/icce.v32.management.37

Ministère de la Transition Ecologique et Solidaire (MTES). (2017). Guide méthodologique appliqué aux sites classés.

VAN DOORSLAER K., DE ROUCK J., BAELUS L., GYSENS S. (2011). (Architectural) Measures to control wave overtopping inside the harbor of Oostende. 5th International Short conference on Applied Coastal Research (SCACR). 